
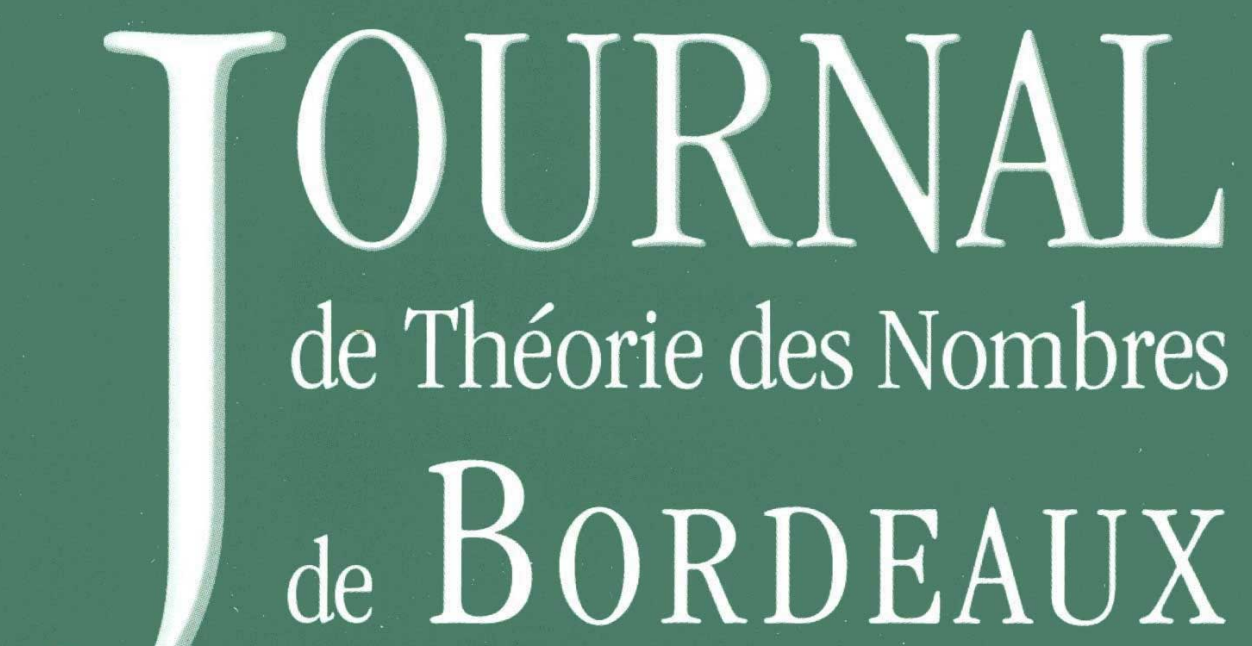

anciennement Séminaire de Théorie des Nombres de Bordeaux

\title{
Gérald TENENBAUM
}

Normal largest gap between prime factors

Tome 31, nº 3 (2019), p. 747-749.

$<$ http://jtnb.centre-mersenne.org/item?id=JTNB_2019__31_3_747_0>

(C) Société Arithmétique de Bordeaux, 2019, tous droits réservés. L'accès aux articles de la revue «Journal de Théorie des Nombres de Bordeaux » (http://jtnb.centre-mersenne.org/), implique l'accord avec les conditions générales d'utilisation (http://jtnb. centre-mersenne.org/legal/). Toute reproduction en tout ou partie de cet article sous quelque forme que ce soit pour tout usage autre que l'utilisation à fin strictement personnelle du copiste est constitutive d'une infraction pénale. Toute copie ou impression de ce fichier doit contenir la présente mention de copyright.

\section{cedram}

Article mis en ligne dans le cadre du

Centre de diffusion des revues académiques de mathématiques

http://www.centre-mersenne.org/ 


\title{
Normal largest gap between prime factors
}

\author{
par GÉRALD TENENBAUM
}

RÉSUMÉ. Désignons par $\left\{p_{j}(n)\right\}_{j=1}^{\omega(n)}$ la suite croissante des facteurs premiers distincts d'un entier $n$. Nous explicitions les détails de la preuve d'un énoncé d'Erdős impliquant que, pour toute fonction $\xi(n)$ tendant vers l'infini avec $n$, nous avons

$$
f(n):=\max _{1 \leqslant j<\omega(n)} \log \left(\frac{\log p_{j+1}(n)}{\log p_{j}(n)}\right)=\log _{3} n+O(\xi(n))
$$

pour presque tout entier $n$.

AbstraCt. Let $\left\{p_{j}(n)\right\}_{j=1}^{\omega(n)}$ denote the increasing sequence of distinct prime factors of an integer $n$. We provide details for the proof of a statement of Erdős implying that, for any function $\xi(n)$ tending to infinity with $n$, we have

$$
f(n):=\max _{1 \leqslant j<\omega(n)} \log \left(\frac{\log p_{j+1}(n)}{\log p_{j}(n)}\right)=\log _{3} n+O(\xi(n))
$$

for almost all integers $n$.

In private correspondence [4], E. Sofos asked for normal upper and lower bounds for the arithmetic function

$$
f(n):=\max _{1 \leqslant j<\omega(n)} \log \left(\frac{\log p_{j+1}(n)}{\log p_{j}(n)}\right),
$$

where $\left\{p_{j}(n)\right\}_{j=1}^{\omega(n)}$ denotes the increasing sequence of distinct prime factors of an integer $n$. The answer to this question is actually provided by a statement of Erdős in [1], where it is asserted without proof that, for any $c>0$, the density of the set of those integers $n$ such that $\mathrm{e}^{f(n)}>c \log _{2} n$ is $1-\mathrm{e}^{-1 / c}$. (Here and in the sequel, $\log _{k}$ denotes the $k$-fold iterated logarithm.) Indeed, given any function $\xi(n)$ tending to infinity and letting $c$ tend sufficiently slowly to zero, we infer that, for almost all $n$, we have $f(n)>\log _{3} n-\xi(n)$, while by letting $c$ tend slowly to infinity we obtain that $f(n) \leqslant \log _{3} n+\xi(n)$ also holds for a set of asymptotic density 1 . Interesting related results appear in Erdös' articles [2] and [3].

In this short note, we provide the not so obvious details of the proof, in the spirit of the paper [1].

Manuscrit reçu le 22 février 2019, accepté le 15 juin 2019

2010 Mathematics Subject Classification. 11N56, 14G42.

Mots-clefs. Distribution of prime factors, normal order, largest gap. 
Theorem 1 (Erdős [1]). Let $c>0$. Then the inequality

$$
f(n)>\log _{3} n-\log (1 / c)
$$

holds on a set of integers $n$ of asymptotic density $1-\mathrm{e}^{-1 / c}$. In particular, given any function $\xi(n) \rightarrow \infty$, we have

$$
\log _{3} n-\xi(n) \leqslant f(n) \leqslant \log _{3} n+\xi(n)
$$

for almost all integers $n$.

Proof. Let $x$ be a large parameter, write $Z:=c \log _{2} x$, and let $\chi_{p}(n)$ denote the indicator function of the set of those integers $n \leqslant x$ that are divisible by the prime $p$ but by no prime $q$ such that $p<q \leqslant p^{Z}$. For squarefree $m$, put $\chi_{m}(n):=\prod_{p \mid m} \chi_{p}(n)$. Taking into account the slow growth of $\log _{2} x$, it is sufficient to show that, as $x$ tends to infinity,

$$
N(x):=\left|\left\{n \leqslant x: p \mid n, p \leqslant x^{1 / Z} \Rightarrow \chi_{p}(n)=0\right\}\right| \sim \mathrm{e}^{-1 / c} x .
$$

Indeed, $n \leqslant x$ is counted by $N(x)$ if, and only if, $p_{j+1}(n) \leqslant p_{j}(n)^{Z}$ whenever $1 \leqslant j<\omega(n)$. Let $P^{+}(n)$ (resp. $P^{-}(n)$ ) denote the largest (resp. the smallest) prime factor of an integer $n$, with the convention that $P^{+}(1)=1$ (resp. $P^{-}(1)=\infty$ ), and let $\mu$ designate the Möbius function. By the inclusion-exclusion principle (see, e.g., [6, p. 39]) or the Möbius inversion formula, we thus have, for $x \geqslant 1$,

$$
N(x)=\sum_{\substack{m \leqslant x \\ m \in \mathcal{M}}} \mu(m) \sum_{n \leqslant x} \chi_{m}(n),
$$

where $\mathcal{M}$ denotes the set of all squarefree integers $m \leqslant x$ such that

$$
P^{+}(m) \leqslant x^{1 / Z} \text {, and } p_{j+1}(m)>p_{j}(m)^{Z} \quad(1 \leqslant j<\omega(m)) .
$$

Moreover, as is familiar in Brun's sieve method, we obtain a lower or an upper bound for the left-hand side by restricting the outer sum to integers $m$ whose number of prime factors is at most equal to an odd or an even bound.

Let $k \geqslant 1$ be fixed. For each $m \in \mathcal{M}$, define $P_{m}:=\prod_{p \mid m} \prod_{p<q \leqslant p^{z}} q$. Then

$$
N_{k}(x):=\sum_{\substack{m \in \mathcal{M} \\ \omega(m)=k}} \sum_{n \leqslant x} \chi_{m}(n)=\sum_{\substack{m \in \mathcal{M} \\ \omega(m)=k}} \sum_{\substack{\nu \leqslant x / m \\\left(\nu, P_{m}\right)=1}} 1 .
$$

From a standard sieve result (see, e.g., the lemma in [5]), we infer that the inner sum is

$$
\sim \frac{x \varphi\left(P_{m}\right)}{m P_{m}}=\frac{x}{m Z^{k}}\left\{1+O\left(\frac{1}{\log P^{-}(m)}\right)+o(1)\right\}
$$

provided $P^{+}(m) \leqslant x^{o(1 / Z)}$. The contribution of the remaining integers $m$ will be treated as an error term. Indeed, in this latter case we may plainly 
assume that $x^{\varepsilon_{x}}<P^{+}(m)^{Z} \leqslant x$, with $\varepsilon_{x}$ tending to 0 arbitrarily slowly. Then the inner sum is classically (see, e.g., [6, Exercise 85])

$$
\ll \frac{x \varphi\left(P_{m}\right)}{m P_{m}} \ll \frac{x}{m Z^{k}}
$$

and the corresponding contribution to $N_{k}(x)$ is

$$
\frac{x}{Z^{k}} \sum_{x^{\varepsilon} x / Z<p \leqslant x^{1 / Z}} \frac{1}{p} \sum_{\substack{P^{+}(h) \leqslant x \\ \omega(h)=k-1}} \frac{1}{h} \ll \frac{x \log \left(1 / \varepsilon_{x}\right)}{\log _{2} x}=o(x) .
$$

Carrying (4) back into (3) and summing over $m$, we obtain, for $k \geqslant 0$,

$$
N_{k}(x)=\frac{x}{Z^{k}} \sum_{\substack{p_{k}^{Z} \leqslant x \\ p_{j}^{Z} \leqslant p_{j+1}(1 \leqslant j<k)}} \frac{1+O\left(1 / \log p_{1}\right)+o(1)}{p_{1} \cdots p_{k}} \sim \frac{x\left(\log _{2} x\right)^{k}}{k ! Z^{k}} \sim \frac{x}{c^{k} k !} .
$$

Thus, for arbirary $\ell \geqslant 1$, we have

$$
x \sum_{0 \leqslant k \leqslant 2 \ell+1} \frac{(-1)^{k}}{c^{k} k !}+o(x) \leqslant N(x) \leqslant x \sum_{0 \leqslant k \leqslant 2 \ell} \frac{(-1)^{k}}{c^{k} k !}+o(x),
$$

and the required result follows by selecting $\ell$ arbitrarily large.

Acknowledgements. The author wishes to express warm thanks to Régis de la Bretèche for valuables discussions on this matter.

\section{References}

[1] P. ERDős, "Some remarks on prime factors of integers", Can. J. Math. 11 (1959), p. 161-167.

[2] - "On some properties of prime factors of integers", Nagoya Math. J. 27 (1966), p. 617-623.

[3] — "On the distribution of prime divisors", Aequationes Math. 2 (1969), p. 177-183.

[4] E. Sofos, private e-mail message, August 30, 2018.

[5] G. Tenenbaum, "Cribler les entiers sans grand facteur premier", Philos. Trans. R. Soc. Lond., Ser. A 345 (1993), p. 377-384.

[6] - Introduction to analytic and probabilistic number theory, 3rd ed., Graduate Studies in Mathematics, vol. 163, American Mathematical Society, 2015.

Gérald Tenenbaum

Institut Élie Cartan

Université de Lorraine

BP 70239

54506 Vandœuvre-lès-Nancy Cedex, France

E-mail: gerald.tenenbaum@univ-lorraine.fr

$U R L$ : http://www.iecl.univ-lorraine.fr/ Gerald.Tenenbaum/ 